\title{
Timing of moderate level prenatal alcohol exposure influences gene expression of sensory processing behavior in rhesus monkeys
}

\author{
Mary L. Schneider ${ }^{1,2,3 *}$, Colleen F. Moore ${ }^{2}$, Julie A. Larson ${ }^{1,3}$, Christina S. Barr ${ }^{4}$, Onofre T. DeJesus ${ }^{5}$ and \\ Andrew D. Roberts ${ }^{6}$
}

1 Department of Kinesiology, University of Wisconsin-Madison, Madison, WI, USA

2 Department of Psychology, University of Wisconsin-Madison, Madison, WI, USA

3 Harlow Center for Biological Psychology, University of Wisconsin-Madison, Madison, WI, USA

4 National Institute on Alcohol Abuse and Alcoholism National Institutes of Health, Bethesda, MD, USA

5 Department of Medical Physics, University of Wisconsin-Madison, Madison, WI, USA

${ }^{6}$ Department of Physics, Minnesota State University, Mankato, MN, USA

\author{
Edited by: \\ John J. Foxe, Nathan S. Kline Institute \\ for Psychiatric Research, USA; City \\ College of the City University of \\ New York, USA \\ Reviewed by: \\ J. N. Reynolds, Queen's University, \\ Canada \\ Pierfilippo De Sanctis, Nathan S. Kline \\ Institute for Psychiatric Research, USA \\ *Correspondence: \\ Mary L. Schneider, Department of \\ Kinesiology, Occupational Therapy \\ Program, University of Wisconsin- \\ Madison, 2175 Medical Science \\ Center, 1300 University Ave. Madison, \\ WI 53706, USA. \\ e-mail: schneider@education.wisc.edu
}

Sensory processing disorder, characterized by over- or under-responsivity to non-noxious environmental stimuli, is a common but poorly understood disorder. We examined the role of prenatal alcohol exposure, serotonin transporter gene polymorphic region variation (rh5-HTTLPR), and striatal dopamine (DA) function on behavioral measures of sensory responsivity to repeated non-noxious sensory stimuli in macaque monkeys. Results indicated that early gestation alcohol exposure induced behavioral under-responsivity to environmental stimuli in monkeys carrying the short (s) rh5-HTTLPR allele compared to both early-exposed monkeys homozygous for the long (/) allele and monkeys from middle-to-late exposed pregnancies and controls, regardless of genotype. Moreover, prenatal timing of alcohol exposure altered the relationship between sensory scores and $\mathrm{DAD}_{2} \mathrm{R}$ availability. In early-exposed monkeys, a positive relationship was shown between sensory scores and $D A D_{2} R$ availability, with low or blunted $D A$ function associated with under-responsive sensory function. The opposite pattern was found for the middle-to-late gestation alcohol-exposed group. These findings raise questions about how the timing of prenatal perturbation and genotype contributes to effects on neural processing and possibly alters neural connections.

Keywords: environment by gene interaction, sensory processing disorder, sensory processing scale for monkeys, genotype, dopaminergic $D_{2} R$ binding, serotonin transporter gene polymorphism, timing of moderate prenatal alcohol exposure, early gestation alcohol exposure

\section{INTRODUCTION}

Sensory processing disorder, a regulatory disorder characterized by atypical under- and/or over-responsivity to non-noxious sensory stimulation (Ayres and Robbins, 1979), is a common but poorly understood disorder. Sensory processing disorders appear to occur in several different syndromes including children who spent time in Eastern European orphanages (Lin et al., 2005), as well as boys with ADHD (Parush et al., 2007). Baranek et al. (2006) reported that compared with typically developing children, $60 \%$ of children with autism had increased sensory symptoms including hyper- and hypo-responsiveness. While there are no comprehensive prevalence studies on sensory processing problems in children with FASD, Jirikowic et al. (2008) reported that "children with FASD were three times more likely to be classified in a clinically significant category on the Short Sensory Profile than peers with typical development".

FASD is probably one of many environmental factors that can induce sensory problems. We previously reported that postnatal lead exposure induced sensory processing disorder in rhesus monkeys (Moore et al., 2008). Also, relevant to this study, Goldsmith et al. (2006) found that tactile and auditory defensiveness showed moderate genetic influences.
In this study we examine the role of temporal aspects of prenatal influences, genotype, and postnatal dopamine (DA) function on sensory processing patterns observed in macaque monkeys. Previous work from several studies in our laboratory found that prenatal alcohol exposure, prenatal stress, and postnatal lead exposure induced deviations from typical sensory processing patterns in monkeys (Moore et al., 2008; Schneider et al., 2008). Along related lines, we found that prenatal alcohol-exposed monkeys carrying the serotonin transporter gene polymorphic region (rh5-HTTLPR) short $(s)$ allele were more irritable and more stress responsive than prenatal alcohol-exposed monkeys homozygous for the long allele or controls regardless of genotype (Kraemer et al., 2008).

We tested the hypothesis that the timing of moderate dose prenatal alcohol exposure would alter sensory responsivity in rhesus monkey offspring as a function of the rh5-HTTLPR length polymorphism. The short 5-HTTLPR results in reduced transcription of the 5-HTT gene which may translate into altered uptake of synaptic serotonin (Lesch et al., 1996). In rhesus macaques (Macaca mulatta), a 21 base pair (bp) insertion/depletion polymorphism (rh5-HTTLPR) that is present in the orthologous region and which is functionally similar to the human 5-HTTLPR variant has been shown to influence transcriptional efficiency (Bennett et al., 2002). Monkeys carrying a 
copy of the rh5-HTTLPR short allele differed in levels of serotonin metabolites in cerebral spinal fluid, neonatal visual orienting to stimuli, and ACTH levels during social separation when compared to monkeys with the $l / l$ genotype. However, these effects were only observed in monkeys exposed to early stress in the form of peer rearing (Bennett et al., 2002; Champoux et al., 2002; Barr et al., 2004).

We assessed behavioral responses to repeated tactile stimuli in macaque monkeys from mothers that were randomly assigned to consume alcohol during early gestation (0-E50), middle-to-late gestation (E50-E135), continuous exposure (0-E135) or controls. Rh5-HTTLPR genotype ( $l / l$ vs. $l / s$ and $s / s$ ) were determined. We also measured striatal DA $\mathrm{D}_{2} \mathrm{R}$ function in positron emission tomography (PET) scans using $\left[{ }^{18} \mathrm{~F}\right]$-fallypride $\left(\left[{ }^{18} \mathrm{~F}\right] \mathrm{FAL}\right)$ developed by Mukherjee et al. (1997). $\left[{ }^{18} \mathrm{~F}\right] \mathrm{FAL}$ is currently the PET tracer of choice for $\mathrm{DA} \mathrm{D}_{2}$ receptor studies because of its optimal imaging properties (Kessler et al., 2009).

We assessed dopaminergic function for several reasons. First, prenatal alcohol exposure has been shown to disrupt the DA system, including DA uptake, receptor binding sites, and size of cell bodies and dendritic growth in DA neurons in rats (Druse et al., 1990; Shetty et al., 1993; Shen et al., 1999). Moreover, frontal-striatal circuitry involved in inhibitory control, executive function and regulatory function, relevant to this study, is dependent on DA and disrupted by fetal alcohol exposure (Kodituwakku et al., 1995; Mattson et al., 1999). We assessed $\mathrm{DA} \mathrm{D}_{2} \mathrm{R}$ availability because the process for assessing this receptor expression is well established from decades of research into neurodegenerative disease such as Parkinson's. The tracer used to assess $\mathrm{DA} \mathrm{D}_{2} \mathrm{R}$ availability was $\left[{ }^{18} \mathrm{~F}\right]$ fallypride (FAL), a fluorine-18-labeled raclopride analogue developed by Mukherjee et al. (1997). FAL has a high affinity for DA $\mathrm{D}_{2}$ receptors and high brain uptake, almost three times higher compared with $\left[{ }^{11} \mathrm{C}\right]$ raclopride.

Our results show that timing of prenatal alcohol exposure interacts with genotype to influence the phenotypical sensory behavior. More specifically, monkeys from early gestation alcohol-exposed groups carrying the short rh5-HTTLPR allele showed lower sensory responsivity scores compared to both alcohol-exposed monkeys homozygous for the long allele and mid-to-late alcohol-exposed monkeys and controls regardless of genotype. Moreover, the relationship between $\mathrm{D}_{2} \mathrm{R}$ availability and sensory responsivity was altered by gestational timing of alcohol such that reduced $\mathrm{D}_{2} \mathrm{R}$ availability was associated with under-responsivity in early alcoholexposed groups and the opposite pattern was found for mid-to-late alcohol-exposed monkeys.

\section{MATERIALS AND METHODS MATERNAL ALCOHOL TREATMENTS}

Healthy adult female rhesus monkeys within the breeding colony that voluntarily and reliably consumed $0.6 \mathrm{~g} / \mathrm{kg}$ of a $6 \%$ volume/volume $(\mathrm{v} / \mathrm{v})$ alcohol solution sweetened with NutraSweet (300 mg/100 ml) (Equal Sweetener, Merisant US, Inc., Chicago, IL, USA) were used in this study. Prior to breeding, blood samples were obtained $60 \mathrm{~min}$ after consumption of $0.6 \mathrm{~g} / \mathrm{kg}$ alcohol, which produced average blood alcohol concentrations of $20-50 \mathrm{mg} / \mathrm{dL}$. This dosage is comparable to an average-size woman consuming two drinks daily. Females willing to consume alcohol prior to breeding were randomly assigned to the control group or one of three experimental groups (see below) with timing of prenatal alcohol exposure as the independent variable. The alcohol-consuming mothers voluntarily consumed the alcohol solution daily at 1600 hours. Water was available ad libitum, including during the period when the alcohol solution was available. The animals had no chow left by the time of day that the alcohol was introduced. The control mothers consumed a sucrose solution that was designed to be approximately equivolemic and equicaloric ( $8 \mathrm{~g} / 100 \mathrm{ml}$ water) to the alcohol solution. All females were housed under identical conditions, undisturbed except for necessary routine animal husbandry. These studies were conducted in accordance with the Institutional Animal Care and Use Committee.

\section{SUBJECTS}

The offspring subjects in this study were 35 male and female rhesus monkeys (Maccaca mulatta), that are members of an ongoing longitudinal study that investigates the effects of moderate level fetal alcohol exposure, during early or mid-late gestation, on brain and neurobehavioral function (see Table 1).

The alcohol exposure periods (Early: days 0 through 50 and Mid-to-Late: 50 through 135) were selected to approximate the embryological and fetal periods in human development, respectively. The embryological periods are extremely similar in rhesus macaques and humans, with major organogenesis essentially complete by approximately day 45 in the rhesus macaque and day 56 in the human (Newell-Morris and Fahrenbruch, 1985). The species differ with regard to the fetal period in that the duration in humans is almost twice that of the macaque. By day 135, the macaque has reached a percentage of brain growth similar to that of a human newborn (Newell-Morris and Fahrenbruch, 1985).

All infant monkeys were housed with their mothers in individual cages during the first 6 months of life. They were separated briefly from their mothers during the first month of life and tested weekly for neonatal neurobehavioral function. At 6 months of age, they were separated permanently from their mothers for weaning. They were then reared in mixed-sex peer groups consisting of 5-6 monkeys from similar prenatal conditions until they were 32 months old. At the time of the neuroimaging studies and sensory testing they were approximately 5 years old, and their housing condition was pair-housing with same-sex peers from similar treatment groups. At that time they were maintained on a diet of Purina Monkey Chow supplemented three times weekly with fresh fruit. All housing conditions were light ( 8 dark and 16 light) and temperature $\left(21 \pm 0.5^{\circ} \mathrm{C}\right)$ controlled.

\section{SENSORY PROCESSING SCALE FOR MONKEYS (SPS-M)}

The SPS-M was developed by adapting procedures from laboratory observational measures of sensory processing for children (Baranek and Berkson, 1994; Miller et al., 1999). Correlations between

Table 1 | Sample size and genotype distribution as a function of prenatal treatment conditions.

\begin{tabular}{lllll}
\hline Genotype & Control & Mid-Late & Early & Continuous \\
\hline II & 6 & 5 & 4 & 5 \\
IS/sS & 4 & 2 & 5 & 4
\end{tabular}


parental reports and the laboratory observational measures of sensory over-responsivity (including tactile defensiveness) have been modest (correlations of .20 to .40) (Baranek and Berkson, 1994). The SPS-M was used in two previous studies with macaque monkeys, one on the effects of moderate level fetal alcohol exposure, alone or in conjunction with prenatal stress (Schneider et al., 2008) and one on the effects of postnatal lead exposure (Moore et al., 2008). Sensory processing testing in this study followed the same procedures as previous studies. Testing was conducted in a $53 \mathrm{~cm} \times 44 \mathrm{~cm}$ testing cage with vertical bars spaced $5.5 \mathrm{~cm}$ apart. The cage was situated in a dimly lit and sound-shielded room $(62 \mathrm{~dB})$ with a masking white noise of $65-70 \mathrm{~dB}$. A human experimenter, who stood beside the cage and administered the tactile items through the bars of the cage, tested each monkey individually. A second experimenter videotaped the session for later scoring. Both experimenters were blind to the experimental conditions of the animals and unfamiliar to the animals.

The first tactile stimulus consisted of a $12.5 \mathrm{~cm}$ feather, which delivered light tactile stimulation. The second stimulus, a $7 \mathrm{~cm}$ cotton ball, delivered a soft but slightly firmer tactile stimulation. Finally, the third stimulus, a $15 \mathrm{~cm}$ stiff craft brush delivered a scratchy but innocuous tactile stimulation. All stimuli were attached to a $91 \mathrm{~cm}$ dowel so the experimenter could maintain a safe distance from the monkey's cage. Six trials of each stimulus were administered in an invariant order, as listed above, as a swipe to the cheek and neck area. Prior to the first presentation of each stimulus, the stimulus was placed in full view and touching range of the monkey and remained there for approximately 3 s. Stimuli were then applied repeatedly to the same side of the animal for approximately $2 \mathrm{~s}$ per trial, with an inter-trial interval of approximately $2 \mathrm{~s}$, and a pause between each of the textures of approximately $4 \mathrm{~s}$. The testing session lasted for approximately $10 \mathrm{~min}$. Raters, blind to the treatment conditions of the animals, scored the videotapes. Each of 18 trials was scored for degree of withdrawal and negative reaction to the tactile stimulus using a 0 to 3 rating scale in 0.25 increments with the integers labeled as follows: 0 = no withdrawal; 1 = slight withdrawal, such as turning head away from the stimulation; 2 = moderate withdrawal, such as turning full body away from stimulation; 3 = extreme withdrawal, such as moving body away from stimulation. Inter-rater reliability as percentage agreement within \pm 0.25 on the rating scale exceeded $99 \%$.

\section{POSITRON EMISSION TOMOGRAPHY (PET) PROCEDURE}

All PET studies were performed as described previously (Roberts et al., 2004; Schneider et al., 2005). Monkeys were fasted overnight and anesthetized with ketamine $(15 \mathrm{mg} / \mathrm{kg}$ ) and transported to the PET facility on campus. Anesthesia during PET scans was maintained with 1.25-2.0\% isoflurane. Each anesthetized animal was positioned in the ECAT 933 PET scanner with horizontal imaging slices parallel to the orbital-meatus (OM) plane. The tracer used to assess $\mathrm{DA} \mathrm{D}_{2}$ receptors was $\left[{ }^{18} \mathrm{~F}\right]$-Fallypride $\left(\left[{ }^{18} \mathrm{~F}\right] \mathrm{FAL}\right)$, an F-18-labeled raclopride analog developed by Mukherjee et al. (1997). $\left[{ }^{18} \mathrm{~F}\right] \mathrm{FAL}$ was prepared using a procedure adopted from Mukherjee et al. (1995). Five $\mathrm{mCi}$ in 1-5 ml normal saline of $\left[{ }^{18} \mathrm{~F}\right] \mathrm{FAL}$ were administered as an intravenous bolus and a dynamic sequence of images over $90 \mathrm{~min}$, including a total of 13 frames with duration increasing from 2 to 10 min was collected. At the end of scanning, the animals were extubated, allowed to awaken, and then returned to the animal care facility. The UW Animal Care and Use Committee approved the protocol that was used in these studies in compliance with NIH regulations on the use of non-human primates in research.

PET images were reconstructed from the raw data using the Ordered Subset Estimation Method (Hudson and Larkin, 1994). Standard regions of interest (ROI) were placed on the occipital cortex (an area known to contain little significant DA-ergic innervation) in order to produce reference region time-activity curves for use as input functions in graphical analysis. Other ROIs were placed to cover both left and right caudate and putamen (jointly referred to as the striatum) in the basal ganglia. For the reversible tracer, FAL, time-activity data for the ROIs were analyzed with the graphical method of Logan et al. (1996). The outcome measure obtained from the Logan plot is the distribution volume ratio (DVR), the ratio of the FAL distribution volume in the target region (striatum) to that in the reference region (occipital cortex). DVR is related to $\mathrm{D}_{2} \mathrm{R}$ availability by the following relationship: $\mathrm{DVR}=[(\mathrm{Bmax} / \mathrm{Kd})+1]$, where Bmax is the mass-specific concentration of available receptors, not occupied by endogenous DA, and $\mathrm{Kd}$ is the receptor-ligand dissociation rate constant.

\section{DNA EXTRACTION AND GENOTYPING}

Blood samples for genotyping were collected when the animals were approximately 6-to 9-years old. DNA was isolated from whole blood using standard extraction methods. Using a protocol modified from that of Lesch et al. (1997), rh5-HTTLPR was amplified from $25 \mathrm{ng}$ of genomic DNA with oligonucleotide primers (stpr5, 5'-GGCGTTGCCGCTCTGAATGC; intl, $5^{\prime}$-CAGGGGAGATCCTGGGAGGG) in $15 \mu \mathrm{l}$ reactions using Platinum Taq and the PCRX Enhancer System kit, according to the manufacturer's protocol (Invitrogen, Carlsbad, CA, USA). Amplifications were performed on a Perkin-Elmer (Fremont, CA, USA) thermocycler (9700) (with one cycle at $96^{\circ} \mathrm{C} / 5 \mathrm{~min}$ followed by 30 cycles of $94^{\circ} \mathrm{C} / 15 \mathrm{~s}, 60^{\circ} \mathrm{C} / 15 \mathrm{~s}, 72^{\circ} \mathrm{C} / 30 \mathrm{~s}$, and a final 3-min extension at $72^{\circ} \mathrm{C}$. Amplicons were separated by electrophoresis on $10 \%$ polyacrylamide gels, and the short (s,398bp) and long (1,419bp) alleles of the rh5-HTTLPR were identified by direct visualization following ethidium-bromide staining.

\section{STATISTICAL ANALYSIS}

The rated response on each trial was the dependent variable in an early alcohol exposure (early, not early) $\times$ late alcohol exposure (late, not late) $\times$ genotype (short carrier, not short $) \times$ sex $(F, M) \times$ texture (feather, cotton, brush) $\times$ trials (6) analysis of variance (ANOVA) with repeated measures on the last two factors. Interactions of genotype $\times$ sex were deleted from the model. There were no significant trials or texture effects or interactions. Therefore, we analyzed the overall mean rated reaction averaged across both trials and textures in a 2 (early) $\times 2$ (late) $\times 2$ (genotype $) \times 2($ sex $)$ ANOVA. Interactions of genotype $\times$ sex were deleted from the model due to small $n$. Post hoc tests were conducted using the Tukey-Kramer method (Keppel and Wickens, 2004). Pearson product-moment correlations were calculated between SPS-M scores and our measure of DA function. 


\section{RESULTS}

\section{OVERALL RESPONSE TO REPEATED TACTILE STIMULATION}

Figure 1 shows that Early alcohol exposure reduced the response to tactile stimuli in monkeys carrying the short rh5-HTTLPR allele by comparison with $l / l$ monkeys and monkeys not exposed to
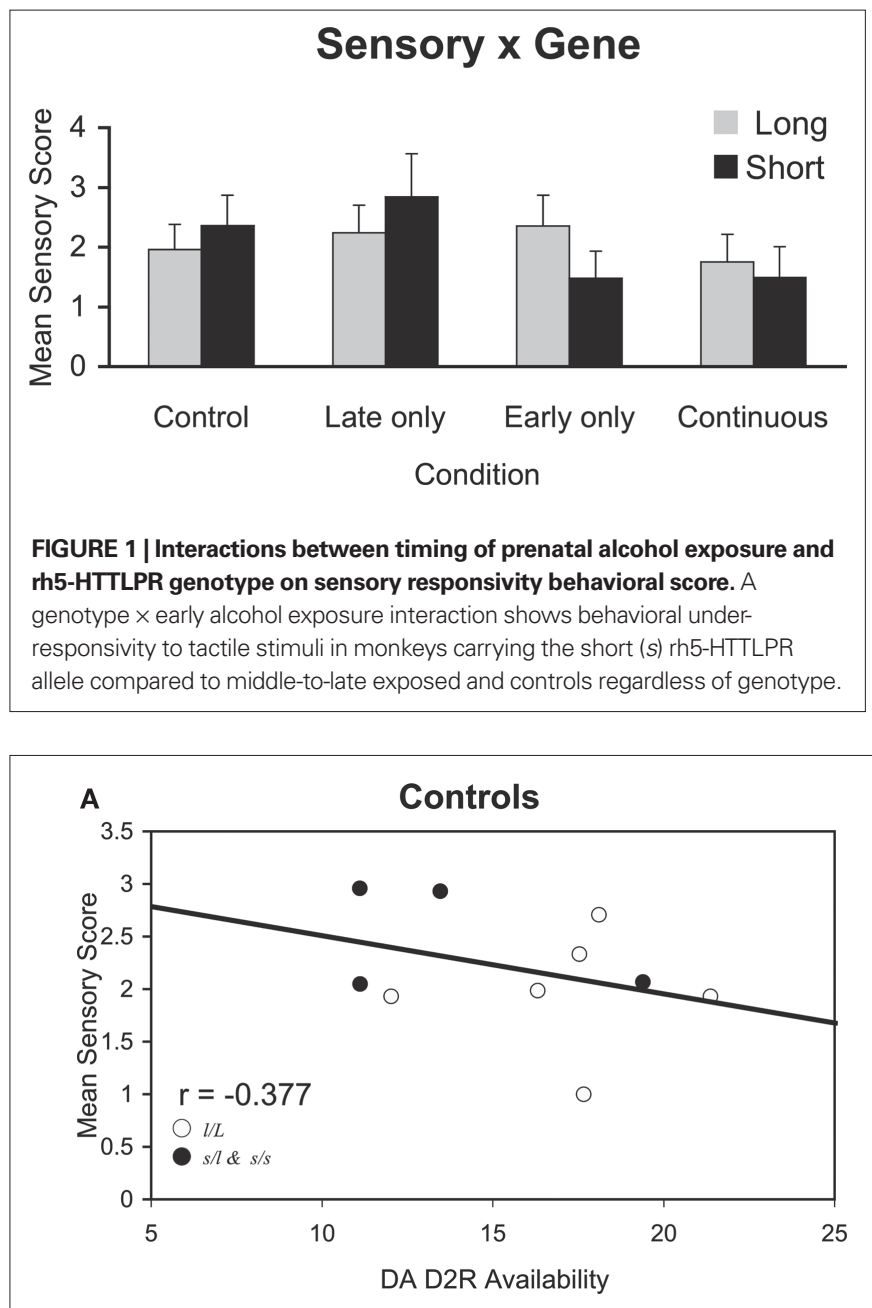

C
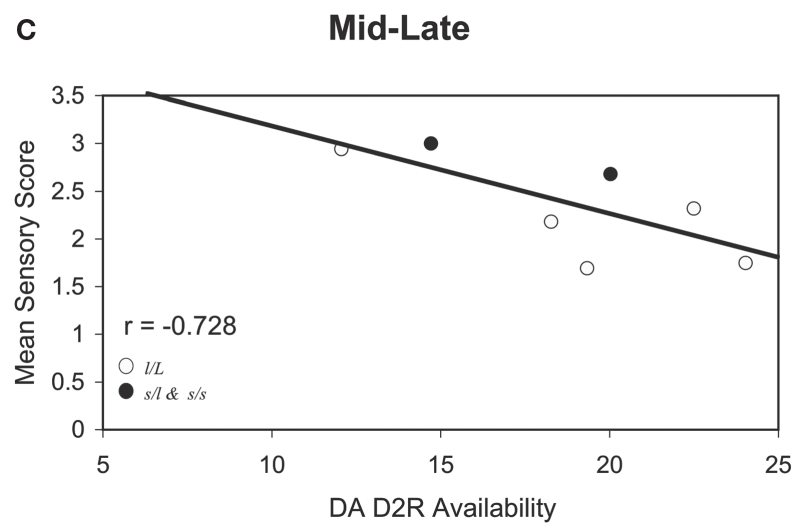

FIGURE 2 | (A-D) Correlations of DA-ergic parameters measured with PET and mean sensory scores in (A) control, (B) early gestation, (C) middle-to-late gestation, and (D) continuous prenatal alcohol-exposed monkeys. This figure illustrates a significant difference in correlations across groups $(p<0.05)$ : alcohol during early gestation carrying the short allele. The 2 (Early alcohol exposure) $\times 2$ (Late alcohol exposure) $\times$ Genotype (Long, Short) $\times$ Sex ANOVA with Sensory Responsivity as the dependent variable revealed a significant Early alcohol $\times$ genotype interaction, $F(1,23)=6.88, p=0.015$. Carriers of the rh5-HTTLPR short allele that were exposed to alcohol during Early gestation (Early (0-E50) and Continuously (0-E135) exposed monkeys) showed the lowest levels of responsiveness to tactile stimuli. Monkeys with the rh5HTTLPR $l / l$ allele did not differ from controls in responsiveness as a function of Early alcohol exposure. A main effect of Early alcohol exposure approached significance, $F(1,23)=2.87, p=0.10$. Sensory responsivity was slightly lower in Early-exposed monkeys than in monkeys not exposed to alcohol during early gestation (Early exposure $M=1.77$, No Early alcohol exposure $M=2.35$ SE's $=0.30$ and 0.27 , respectively).

\section{CORRELATION OF SENSORY SCORES WITH PET MEASURES OF DA-ergic FUNCTION}

Figure 2 displays the correlations of sensory scores with PET measures separately by treatment groups. Over all animals, there was no relationship between sensory scores and $\mathrm{DA}_{2} \mathrm{R}$ availability, $r=0.08$, ns. However, a test for difference of the regression slopes across groups was significant, $p<0.05$. Sensory score correlated

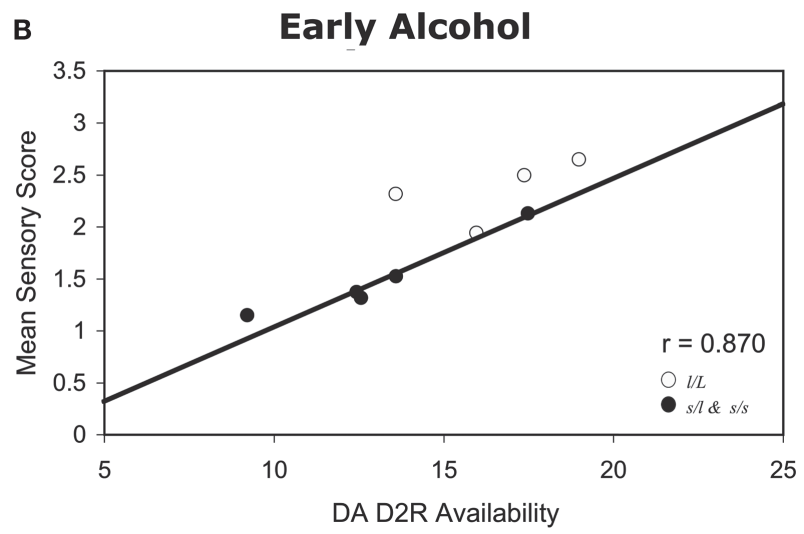

D

Continuous

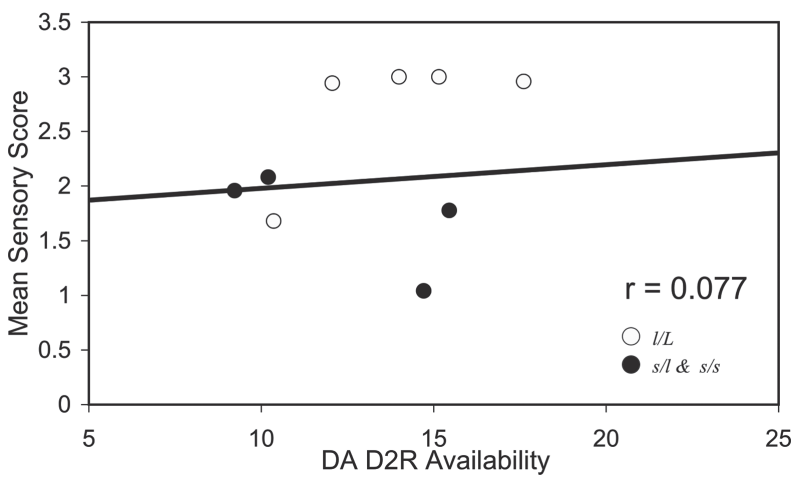

$r=0.87 p<0.01$ for (B) early gestation prenatal alcohol-exposed monkeys compared to $r=-0.728, p<0.10$ for $(\mathbf{C})$ middle-to-late gestation exposed monkeys. Closed circles represent monkeys carrying the short (s) allele and open circles are monkeys with the $/ / /$ allele. 
positively with PET measures of striatal DA $\mathrm{D}_{2} \mathrm{R}$ availability for the Early alcohol-exposed monkeys $(r=0.87, p<0.01)$ whereas sensory scores tended to correlate negatively with striatal $\mathrm{DA} \mathrm{D}_{2} \mathrm{R}$ availability for the Mid-late alcohol-exposed monkeys $(r=-0.728$, $p<0.10)$. There were no significant correlations between sensory scores and PET FAL binding for the controls $(r=-0.38)$ or for the Continuous-exposed $(r=0.077)$ groups.

\section{DISCUSSION}

We measured behavioral responses to non-noxious tactile stimulation, rh5-HTTLPR genotype, and striatal DA $\mathrm{D}_{2} \mathrm{R}$ availability in macaque monkeys to address the question of whether patterns of sensory responsivity are associated with timing of prenatal alcohol exposure, rh5-HTTLPR genotype, and striatal DA D 2 R availability. There were two principal findings of the present study. First, our results demonstrated that the timing of prenatal alcohol exposure affected the behavioral responsivity of monkeys to sensory stimuli and that the response depended upon both genotype and timing of alcohol exposure. Our second finding was that the timing of prenatal alcohol exposure also influenced the relationship between sensory processing behavior and striatal DA $D_{2} \mathrm{R}$ availability. Here we consider the potential mechanisms which underlie these results as well as their functional implications.

\section{GENE $\times$ ENVIRONMENT EFFECT}

Our first finding is that the monkeys exposed to alcohol during early gestation (early-exposed, 0-E50, and continuous-exposed, 0-E135) and carrying the short rh5-HTTLPR allele showed reduced sensory responsivity to tactile stimuli compared to other groups. This finding confirms and extends our previous findings that moderate level prenatal alcohol exposure interacts with the short allele of the promoter region of the serotonin transporter gene to alter behavioral outcomes in rhesus monkeys (Kraemer et al., 2008). Thus, the effect of genotype on response to sensory stimuli depends upon whether they were exposed to alcohol during early gestation.

The most likely mechanism is interference with neurodevelopmental processes occurring during early gestation. Further, our findings suggest that the monkeys carrying the short rh5-HTTLPR allele may be more vulnerable to early gestation disruption of neurodevelopmental processes. Three broad phases of brain development have been described in rhesus macaques. These phases are: neurogenesis, neuronal migration, and synaptogenesis (Rakic, 1988). According to Rakic, in both humans and monkeys, cortical neurons are generated near the surface of the cerebral ventricle during early gestation (0-E40). Following their last division, postmitotic cells generated within the proliferation zone migrate along radial glial fascicles and enter the developing cortical plate and form ontogenetic columns (E40-E70.100) (Rakic, 1995). By day E112, the developing cortex has its full complement of neurons. At day E112, the phase of rapid synaptogenesis begins. This phase has been shown to occur synchronously in the somatosensory, motor, and associative areas (Zecevic and Rakic, 1991) and continues to the third month postnatally (Bourgeois and Rakic, 1993).

Our present findings advance our understanding of the timing of prenatal alcohol exposure on sensory responsivity by demonstrating that, in monkeys carrying the rh5-HTTLPR $(s)$ allele, exposure to a teratogen during neurogenesis (0-E40) differs from exposure during other times. Studies have found that exposure to teratogens during the period of neurogenesis in early gestation can induce gross irreversible malformations of the brain. Some have found that fetal alcohol exposure during early pregnancy, or neurogenesis, is equally or most damaging to fetal CNS development and function, than exposure during other periods, including craniofacial anomalies, midline brain abnormalities, and reduced cerebellar Purkinje cell number in humans, sheep, and rodents (Sulik et al., 1981; Graham et al., 1988; Ramadoss et al., 2007). This does not mean that later prenatal perturbations are inconsequential. Alcohol exposure during the latter part of pregnancy has been shown to be more strongly associated with developmental problems in some human studies (Richardson et al., 1989; Coles et al., 1991; Cornelius et al., 1999; Day et al., 1999). Moreover, in rodents, exposure to alcohol during the postnatal period of high synaptogenesis (second and third trimester equivalent in humans) produced neuronal loss, altered neuronal circuitry, and altered gliosis and apoptotic neurodegeneration in the developing forebrain (Borges and Lewis, 1983; West et al., 1984; Guerri, 1987; Bonthius and West, 1991; Goodlett et al., 1993, 1998; Melcer et al., 1994).

Studies have shown that the mid-gestational period of neuronal migration is also highly sensitive to various insults, including toxins, viruses, and genetic mutations (Rakic, 1988). Abnormal neuronal migration is considered to cause both gross and subtle synaptic circuit abnormalities (Barth, 1987; Rakic, 1988; Caviness et al., 1989), including developmental dyslexia (Galaburuda et al., 1989) and schizophrenia (Kotrla et al., 1997). Correct cell migration is important for communication between early- and later-forming neurons at critical developmental stages prior to making synaptic connections (Rakic, 1985). Correct neural migration is important for the appropriate neuron position, which subsequently impacts brain morphology and function.

It is interesting to note that the effects of prenatal alcohol exposure on sensory processing function in subjects in the continuous group (0-E135) carrying the short allele are equivalent to those with the early exposure group (0-E50) short allele carriers, in contrast to those from the middle-to-late exposed group (E50-E135) carrying the $(s)$ allele. This suggests that, for monkeys carrying the short allele, if the nervous system was exposed to alcohol during early gestation, then middle-to-late exposure (in the continuous group) induces different effects on sensory processing function relative to middle-to-late exposure alone. In other words, this study shows that the effects of prenatal alcohol exposure during middle-to-late gestation are dependent upon events that occur during early gestation in the continuous-exposed group. Early exposure appears to have altered the nervous system such that it responds to middle-to-late exposure in a different way. Others have reported similar findings. For example, in rats, alcohol exposure during E14 and E15 lowered the number of cells in the ventral lateral nucleus of the thalamus, whereas alcohol exposure E11-E20 did not, suggesting that the timing of the initiation and duration of exposure is critical (Livy et al., 2001).

The finding that the timing of alcohol exposure interacted with genotype to alter sensory responsivity is a novel and a potentially important finding. The most likely mechanism underlying this interaction is that rh5-HTTLPR genotype can influence the early 
patterning of the brain and impact how alcohol exposure induces brain changes at that time. Studies in mice have shown that carrying the short version can affect neurite growth, migration, and synaptogenesis. (Lesch et al., 1997; Murphy et al., 2004) and in humans, the 5-HTTLPR s allele has been linked to anxiety-related personality traits in some studies (Lesch et al., 1996; Hamer et al., 1999). It has also been demonstrated that 5-HTTLPR can interact with various environmental stressors to increase risk for psychopathology (Caspi et al., 2003). In monkeys reared without mothers (a model of early adversity, which produces long-lasting effects on brain structure and behavioral/endocrine stress responding), the $s$ allele predicts CSF levels of the serotonin metabolite 5-HIAA, neonatal behavior, and stress responsivity (Bennett et al., 2002; Champoux et al., 2002; Barr et al., 2004). It, therefore, is likely that HTTLPR genotype might interact with other types of stressors that impact brain development.

Variation in genotypes can play a role in 5-HT neurotransmission by impacting the uptake of 5-HT into the presynaptic neuron (Glatz et al., 2003). 5-HT is an important modulator of early CNS development, including cell proliferation, migration and differentiation (Lauder, 1990, 1993). 5-HT acts as a master control neurotransmitter, regulating several other neurotransmitters. Because early gestation is a critical developmental period during which time neurons are being generated, genetic variation that impacts 5-HT system function during early embryonic/fetal development might render an organism more or less sensitive to prenatal alcohol exposure. Genetic effects on sensitivities to teratogenic conditions (including alcohol exposure) might be a predictive response for adaptation to environmental conditions present at the time of birth, presumably also with environment risks, promoting better opportunities for survival. That is, prenatal and postnatal environmental risks may be correlated. In this case, genes might serve as a protective function in relation to environmental insults. At the same time, this variability might render some offspring vulnerable or less well suited to adapt to, or even survive in a hazardous environment. Interpreted within the context of an evolutionary concept of natural selection, variation in an organism's response to the environment is important and serves as the basic ingredient for natural selection (Ridley, 2003). It is important for an organism to adapt to its environment and such adaptation during the prenatal period makes sense, given this is the formative period of development. Thus, one can assume that one genetic variant versus another might render the organism more or less sensitive to certain environmental conditions occurring at the particular formative time of development. This process could ultimately provide an organism with a better fit with the environment and thus promote positive outcomes.

It is interesting that there were no main effects of genetic variant of the promoter region of the serotonin transporter gene on sensory processing function in this study. This is consistent with the notion that susceptibility genes for multifactorial psychological disorders have very negligible effects by themselves, but can increase risk when interacting with other gene variants and environmental factors (Kendler, 2005). The idea is that susceptibility genes represent particular allelic variations of common genes or normal allelic variation. Thus the genes may affect physiological pathways that could render psychological disorders more or less inevitable, but the genes do not cause the disorder directly (Rutter et al., 2006). Rather they contribute to genetically influenced sensitivities to specific environments.

\section{RELATIONSHIP BETWEEN PET DA MEASURES AND BEHAVIORAL MEASURES OF SENSORY RESPONSIVITY}

We also found that the timing of gestational exposure to alcohol affected the relationship between behavioral measures of sensory responsivity and PET measures of DA-ergic function. For the early alcohol-exposed monkeys, there was a positive relationship between sensory responsivity and striatal $\mathrm{DA} \mathrm{D}_{2} \mathrm{R}$ availability. In other words, for early-exposed monkeys low levels of striatal DA $D_{2} R$ availability, which is suggestive of blunted DA function (Volkow et al., 2002), were related to sensory under-responsivity. The opposite pattern was found for middle-to-late exposed monkeys, in that low $\mathrm{D}_{2} \mathrm{R}$ availability was associated with high sensory responsivity.

It is interesting that our previous work showed that timing of alcohol exposure disrupted striatal DA system function in different ways (Schneider et al., 2005). Overall, striatal DA D R density was decreased in Early-exposed monkeys (Early only and Continuous exposed) compared to monkeys not exposed to alcohol during early gestation (Controls and Middle-to- late exposed) (Schneider et al., 2005). This finding underscores the notion that exposure during early gestation (0-E50), approximating the period of neurogenesis (0-E40) differs substantially from exposure during middle-tolate gestation (E50-E135), approximating the period of neuronal migration (E40-E70/100) and early synaptogensis (E112-third month postnatally (Rakic, 1988). Either an increase or decrease in DA function can have repercussions on normal functioning. It has been shown that there is a critical range of DA activity for optimal functioning (Arnsten, 1997).

DA is an important neurotransmitter, modulating activity in many brain regions, promoting both excitatory and inhibitory signals. The striatal region has a high density of $\mathrm{D}_{2} \mathrm{R}$ which are considered to have a gating function in basal ganglia thalamocortical circuits thought to underlie inhibitory control (Casey, 2001). DA is considered to underlie the behavioral responses to important or salient events, whether aversive or appetitive (Berridge and Robinson, 1998; Redgrave et al., 1999). If DA $\mathrm{D}_{2} \mathrm{R}$ density is low or activity blunted, it is thought that attention, motor function, or executive function could be disrupted. For example, DA $\mathrm{D}_{2} \mathrm{R}$ deficient mice need more trials to alter responses during reversal testing than wild-type mice, suggesting $\mathrm{DA} \mathrm{D}_{2} \mathrm{R}$ simulation in striatum may brake or reduce excitatory corticostriatal signaling so that goal-directed behavior can be guided by signaling the organism about contingency changes (Carlsson et al., 2001). If DA $\mathrm{D}_{2} \mathrm{R}$ is too high, sensitivity to novel stimuli is considered to be heightened. It has also been suggested that reduced striatal $\mathrm{D}_{2} \mathrm{R}$ density could reduce sensitivity to hedonic positive stimuli, increasing the compulsive drive to consume alcohol or drugs (Volkow et al., 2002). Disruptions in DA-ergic transmission contribute to neuropsychiatric disorders, including schizophrenia and drug addiction (Bergman et al., 1998; Berke and Hyman, 2000). The nature of DA-mediated effects depends on DA receptor subtype as well as other factors. Unmedicated patients with schizophrenia have been found to have higher striatal $\mathrm{D}_{2} \mathrm{R}$ density 
compared to normal controls (Laruelle, 1998). It could be hypothesized that the timing of the alcohol exposure could alter the relationship between DA-ergic function and sensory sensitivity to environmental stimuli by altering one or more of the processes of neurogenesis, cell proliferation, migration or synaptogenesis leading to possible mis-wiring of neural processes and consequent disrupted behavioral effects.

It was surprising to find that the continuously exposed group, consisting of both early and mid-late exposure and hence involving a longer duration of exposure, showed a different relationship between $\mathrm{D}_{2} \mathrm{R}$ availability and SPS-M scores than that of the early or mid-late exposed groups. However, findings such as these are not unprecedented in the literature. The way in which prenatal alcohol exposure impedes normal brain development remains unclear. Alcohol has wide effects on the brain, neurotransmitter systems, and behavior - possibly affecting a number of developmental processes concurrently. These effects differ by timing of exposure, region and cell type. Interestingly, and relevant to our findings, some areas of the brain may be differentially affected by longer durations than shorter exposures to alcohol (Sulik et al., 1981; Livy et al., 2001). For example, Livy et al. (2001) found that in rats, alcohol exposure during E14 and E15 resulted in lower numbers of cells in the ventral lateral nucleus of the thalamus but that alcohol exposure during E11 to E20 did not produce this effect. They speculated that the timing of alcohol exposure initiation could be an important influence on the effects of alcohol exposure. It has also been suggested that the longer period of treatment might afford the opportunity to habituate to the treatment and possibly to develop tolerance (Smith et al., 1989). While it is unclear how this occurs at this time, there is some evidence to support the notion that, in some cases, shorter durations of exposure may induce adverse effects not observed with longer periods of exposure.

Also relevant to our findings, Clarren et al. (1990) found that, in monkeys, a high alcohol exposure condition induced a negative correlation between offspring striatal DA and maternal blood alcohol concentration whereas in a moderate alcohol condition, the relationship between maternal blood alcohol concentration and offspring straital DA was positive. More research is clearly needed to understand how the intensity, duration, and timing of alcohol exposure induces brain and related behavioral changes and in affected offspring.

An important idea related to these findings concerns the notions of equifinality and multifinality, or that there are multiple pathways to adaptive and maladaptive outcomes and diverse outcomes from individual risk factors (Cicchetti and Rogosch, 1996). For example, in this study, while exposure to early gestation alcohol yielded a positive relationship between DA-ergic function and sensory responsivity, exposure during middle-to-late resulted in the opposite pattern, suggesting that there are multiple outcomes for individuals, despite similar exposure to environmental risk factors, such as prenatal alcohol exposure. Thus one would expect a range of patterns of responsivity to sensory stimuli in children with fetal alcohol spectrum disorder (FASD), depending upon a variety of variables including the timing of exposure and genotype. Indeed, children with FASD were rated by parents as deficient in visualspatial, auditory, and tactile processing (Morse and Cermak, 1994). One would also expect that sensory processing disorder would be associated with behavioral abnormalities in children especially related to inhibitory control. Indeed studies have shown that children with fetal alcohol spectrum disorder who demonstrate sensory processing deficits demonstrate externalizing problems, difficulties in attention, socialization, and rule breaking as well as thought problems (Franklin et al., 2008; Jirikowic et al., 2008).

Another interesting implication is that early gestation alcohol exposure, a time at which pregnancy might not yet be detected, is a vulnerable time, especially for those individuals carrying genetic vulnerability factors. Since both under-and over-responsivity to sensory stimuli are associated with behavioral problems, the possibility of different etiology and mechanisms must be taken seriously. Perhaps prenatal alcohol exposure induces a range of alterations in the generation, proliferation and migration of cells which could then promote deviations in behavior, depending upon multiple factors, including timing of exposure to environmental perturbations and genotype.

There are several limitations of this study. One is that the candidate gene was limited to one functional 5-HTT marker (vs. haplotype). Moreover, candidate loci that affect functioning of other neurotransmitter systems are undoubtedly important and relevant to contributing to behavioral disorders in fetal alcohol-exposed individuals. Another limitation is that we only assessed one neurotransmitter subtype in this study, $\mathrm{D}_{2} \mathrm{R}$, and we were limited to the striatum brain region. We are currently expanding our studies to assess $\mathrm{D}_{1}$-type receptors and DA transporters in multiple brain regions in these monkeys. Also, while experiments with non-human primates permit control of the dose and timing of prenatal alcohol exposure and control of potential co-variants, behavioral measures that are relevant to humans and candidate genes and PET measures of brain function similar to humans, caution is needed in generalizing from monkeys to humans. Thus, it is critical to replicate these findings in human studies. Another limitation is that mothers were pre-selected for consuming a moderate dose of alcohol. Whether this involves genetic taste preference or susceptibility to alcoholinduced effects is unknown. Finally, if these results replicate in humans, treatment studies are critically needed to determine if reduced sensory sensitivity can be reversed in infants and children with genetic vulnerabilities and exposure to alcohol during early gestation using sensory-based occupational therapy methodology (Baranek, 2002; Miller et al., 2007; Schaaf and Nightlinger, 2007). If such individuals can be identified early in life, intervention studies are possible early in life at the peak of brain plasticity. These studies have the potential to significantly reduce the pain and suffering associated with the devastating effects of fetal alcohol spectrum disorder.

In summary, there is clear evidence from rat studies that prenatal alcohol exposure effects are related to the region of the brain or cell type undergoing rapid development at that time (Goodlett and Johnson, 1999). Our findings provide evidence in primates that genetic factors might increase or decrease an organism's sensitivity to fetal alcohol effects during a particular gestation period.

\section{ACKNOWLEDGMENT}

This study was supported by AA10079 and AA12277 from the National Institute of Alcoholism and Alcohol Abuse and Wallace Research Foundation Grant to Mary L. Schneider. 


\section{REFERENCES}

Arnsten, A. F. T. (1997). Catecholamine regulation of the prefrontal cortex. J. Psychopharmacol. (Oxford) 11, 151-162.

Ayres, J.A., and Robbins, J. (1979). Sensory Integration and the Child. Los Angeles, Western Psychological Services.

Baranek, G. T. (2002). Efficacy of sensory and motor interventions for children with autism. J. Autism Dev. Disord. 32, 397-422.

Baranek, G. T., and Berkson, G. (1994). Tactile defensiveness in children with developmental disabilities: responsiveness and habituation. J. Autism Dev. Disord. 24, 457-471.

Baranek, G. T., David, F. J., Poe, M. D., Stone, W. L., and Watson, L. R. (2006). Sensory Experiences Questionnaire: discriminating sensory features in young children with autism, developmental delays, and typical development. J. Child. Psychol. Psychiatry 47, 591-601.

Barr, C. S., Newman, T. K., Shannon, C., Parker, C., Dvoskin, R. L., Becker, M. L., Schwandt, M., Champoux, M., Lesch, K. P., Goldman, D., Suomi, S. J., and Higley, J. D. (2004). Rearing condition and rh5-HTTLPR interact to influence limbic-hypothalamic-pituitary-adrenal axis response to stress in infant macaques. Biol. Psychiatry 55, 733-738.

Barth, P. G. (1987). Disorders of neuronal migration. Can. J. Neurol. Sci. 14, $1-16$.

Bennett, A. J., Lesch, K. P., Heils, A., Long, J. C.,Lorenz,J.G.,Shoaf, S. E.,Champoux, M., Suomi, S. J., Linnoila, M. V., and Higley, J. D. (2002). Early experience and serotonin transporter gene variation interact to influence primate CNS function. Mol. Psychiatry 7, 118-122.

Bergman,H., Feingold,A., Nini,A., Raz, A., Slovin, H., Abeles, M., and Vaadia, E. (1998). Physiological aspects of information processing in the basal ganglia of normal and parkinsonian primates. Trends Neurosci. 21, 32-38.

Berke, J. D., and Hyman, S. E. (2000). Addiction, dopamine, and the molecular mechanisms of memory. Neuron 25, 515-532.

Berridge, K. C., and Robinson, T. E. (1998). What is the role of dopamine in reward: hedonic impact, reward learning, or incentive salience? Brain Res. Brain Res. Rev. 28, 309-369.

Bonthius, D. J., and West, J. R. (1991). Permanent neuronal deficits in rats exposed to alcohol during the brain growth spurt. Teratology 44, 147-163.

Borges, S., and Lewis, P. D. (1983). Effects of ethanol on postnatal cell acquisition in the rat cerebellum. Brain Res. 271, 388-391.

Bourgeois, J. P., and Rakic, P. (1993). Changes of synaptic density in the primary visual cortex of the macaque monkey from fetal to adult stage. J. Neurosci. 13, 2801-2820.

Carlsson, A., Waters, N., HolmWaters, S., Tedroff, J., Nilsson, M., and Carlsson, M. L. (2001). Interactions between monoamines, glutamate, and GABA in schizophrenia: new evidence. Annu. Rev. Pharmacol. Toxicol. 41, 237-260

Casey, B. J. (2001). Disruption of inhibitory control in developmental disorders: a mechanistic model of implicated fronto-striatal circuitry. In Mechanisms of Cognitive Development: Behavioral and Neural Perspectives, J. L. McClelland and R.S. Siegler, eds (Mahwah, NJ, Erlbaum), pp. 327-349.

Caspi, A., Sugden, K., Moffitt, T. E., Taylor, A., Craig, I.W., Harrington, H., McClay, J., Mill, J., Martin, J., Braithwaite, A., and Poulton, R. (2003). Influence of life stress on depression: moderation by a polymorphism in the 5 -HTT gene. Science 301, 386-389.

Caviness, V. S., Misson, J. P., and Gadisseux, J. F. (1989). Abnormal neuronal migrational patterns and disorders of neocortical development. In From Reading to Neuron, A. M. Galaburuda, ed. (Cambridge, MIT Press), pp. 405-442.

Champoux, M., Bennett, A., Shannon, C., Higley, J. D., Lesch, K. P., and Suomi, S. J. (2002). Serotonin transporter gene polymorphism, differential early rearing, and behavior in rhesus monkey neonates. Mol. Psychiatry 7, 1058-1063.

Cicchetti, D., and Rogosch, F. A. (1996). Equifinality and multifinality in developmental psychopathology. Dev. Psychopathol. 8, 597-600.

Clarren, S. K., Astley, S. J., Bowden, D. M., Lai, H., Milam, A. H., Rudeen, P. K., and Shoemaker, W. J. (1990). Neuroanatomic and neurochemical abnormalities in nonhuman primate infants exposed weekly to doses of ethanol during gestation. Alcohol. Clin. Exp. Res. 14, 674-683.

Coles, C. D., Brown, R. T., Smith, I. E., Platzman, K. A., Erickson, S., and Falek, A. (1991). Effects of prenatal alcohol exposure at school age: I. Physical and cognitive development. Neurotoxicol. Teratol. 13, 357-367.

Cornelius, M. D., Goldschmidt, L., Taylor, P. M., and Day, N. L. (1999). Prenatal alcohol use among teenagers: Effects on neonatal outcomes. Alcohol. Clin. Exp. Res. 23, 1238-1244.
Day, N. L., Zuo, Y., Richardson, G. A., Goldschmidt, L., Larkby, C. A., and Cornelius, M. D. (1999). Prenatal alcohol use and offspring size at 10 years of age. Alcohol. Clin. Exp. Res. 23, 863-869.

Druse, M. J., Tajuddin, N., Kuo, A. P., and Connerty, M. (1990). Effects of in utero ethanol exposure on the developing dopaminergic system in rats. J. Neurosci. Res. 27, 233-240.

Franklin, L., Deitz, J., Jirikowic, T., and Astley, S. (2008). Children with fetal alcohol spectrum disorders: problem behaviors and sensory processing. Am J. Occup. Ther. 62, 265-273.

Galaburuda, A. M., Rosen, G. D., and Sherman, G. F. (1989). The neural origin of developmental dyslexia: Implications for medicine, neurology, and cognition. In From Reading to Neurons, A. M. Galaburuda, ed. (Cambridge, MA, MIT Press), pp. 377-404.

Glatz, K., Mossner, R., Heils, A., and Lesch, K.P. (2003). Glucocorticoid-regulated human serotonin transporter (5-HTT) expression is modulated by the 5 -HTT gene-promotor-linked polymorphic region. J. Neurochem. 86, 1072-1078.

Goldsmith, H. H., Van Hulle, C. A., Arneson, C. L., Schreiber, J. E., and Gernsbacher, M. A. (2006). A population-based twin study of parentally reported tactile and auditory defensiveness in young children. J. Abnorm. Child. Psychol. 34, 393-407.

Goodlett, C. R., and Johnson, T. B. (1999). Temporal windows of vulnerability within the third trimester equivalent: why "knowing when" matters. In Alcohol and Alcoholism: Effects on Brain and Development, J. H. Hannigan, C. R. Goodlett, L. P. Spear and N. E. Spear, eds (Mahwah, Earlbaum Associates), pp. 59-91.

Goodlett, C.R., Leo, J.T., O'Callaghan, J. P., Mahoney, J. C., and West, J. R. (1993). Transient cortical astrogliosis induced by alcohol exposure during the neonatal brain growth spurt in rats. Dev Brain Res. 72, 85-97.

Goodlett, C. R., Pearlman, A. D., and Lundahl, K. R. (1998). Binge neonatal alcohol intubations induce dosedependent loss of Purkinje cells. Neurotoxicol. Teratol. 20, 285-292.

Graham, J. M. Jr., Hanson, J. W., Darby, B. L., Barr, H. M., and Streissguth, A. P. (1988). Independent dysmorphology evaluations at birth and 4 years of age for children exposed to varying amounts of alcohol in utero. Pediatrics $81,772-778$.

Guerri, C. (1987). Synaptic membrane alterations in rats exposed to alcohol. Alcohol. Alcohol Suppl. 1, 467-472.
Hamer, D. H., Greenberg, B. D., Sabol, S. Z., and Murphy, D. L. (1999). Role of the serotonin transporter gene in temperament and character. J. Pers. Disord. 13, 312-327.

Hudson, H. M., and Larkin, R. S. (1994). Accelerated image reconstruction using ordered subsets of projection data. IEEE Trans. Med. Imaging 13, 601-609.

Jirikowic, T., Olson, H. C., and Kartin, D. (2008). Sensory processing, school performance, and adaptive behavior of young school-age children with fetal alcohol spectrum disorders. Phys. Occup. Ther. Pediatr. 28, 117-136.

Kendler, K. S. (2005). “A gene for...”: the nature of gene action in psychiatric disorders. Am. J. Psychiatry 162, 1243-1252.

Keppel, G., and Wickens, T. D. (2004). Design and Analysis: A Researcher's Handbook. Upper Saddle River, Pearson Prentice Hall.

Kessler, R. M., Woodward, N. D., Riccardi, P., Li, R., Ansari, M. S., Anderson, S. Dawant, B., Zald, D., and Meltzer, H. Y. (2009). Dopamine D2 receptor levels in striatum, thalamus, substantia nigra, limbic regions, and cortex in schizophrenic subjects. Biol. Psychiatry 65, 1024-1031.

Kodituwakku, P. W., Handmaker, N. S., Cutler, S. K., Weathersby, E. K., and Handmaker, S. D. (1995). Specific impairment in self-regulation in children exposed to alcohol prenatally. Alcohol. Clin. Exp. Res. 19, 1558-1564.

Kotrla, K. J., Sater, A. K., and Weinberger, D. R. (1997). Neuropathology, neurodevelopment and schizophrenia. In Neurodevelopment and Adult Psychopathology, M. S. Keshavan and R. M. Murray, eds (Cambridge, Cambridge University Press), pp. 187-198.

Kraemer, G. W., Moore, C. F., Newman, T. K., Barr, C. S., and Schneider, M. L. (2008). Moderate level fetal alcohol exposure and serotonin transporter gene promoter polymorphism affect neonatal temperament and limbichypothalamic-pituitary-adrenal axis regulation in monkeys. Biol. Psychiatry 63, 317-324.

Laruelle, M. (1998). Imaging dopamine transmission in schizophrenia: a review and meta-analysis. Q. J. Nucl. Med. 42, 211-221.

Lauder, J. M. (1990). Ontogeny of the serotonergic system in the rat: serotonin as a developmental signal. Ann. N. Y. Acad. Sci. 600, 297-313; discussion 314 .

Lauder, J. M. (1993). Neurotransmitters as growth regulatory signals: role of 
receptors and second messengers. Trends Neurosci. 16, 233-240.

Lesch,K.P., Bengel,D.,Heils,A.,Sabol, S. Z., Greenberg, B. D., Petri, S., Benjamin, J., Muller, C. R., Hamer, D. H., and Murphy, D. L. (1996). Association of anxiety-related traits with a polymorphism in the serotonin transporter gene regulatory region. Science 274, 1527-1531.

Lesch, K. P., Meyer, J., Glatz, K., Flugge, G., Hinney, A., Hebebrand, J., Klauck, S. M., Poustka, A., Poustka, F., Bengel, R., Mössner, P., Riederer, P., and Heils, A. (1997). The 5-HT transporter gene-linked polymorphic region (5HTTLPR) in evolutionary perspective: alternative biallelic variation in rhesus monkeys. Rapid communication. J. Neural. Transm. 104, 1259-1266.

Lin, S. H., Cermak, S., Coster, W. J., and Miller, L. (2005). The relation between length of institutionalization and sensory integration in children adopted from Eastern Europe. Am. J. Occup. Ther. 59, 139-147.

Livy, D. J., Maier, S. E., and West, J. R. (2001). Fetal alcohol exposure and temporal vulnerability: Effects of binge-like alcohol exposure on the ventro-lateral nucleus of the thalamus. Alcohol. Clin. Exp. Res. 25, 774-780.

Logan, J., Fowler, J.S., Volkow, N. D., Wang, G. J., Ding, Y. S., and Alexoff, D. L. (1996). Distribution volume ratios without blood sampling from graphical analysis of PET data. J. Cereb. Blood Flow Metab. 16, 834-840.

Mattson, S. N., Goodman, A. M., Caine, C., Delis, D. C., and Riley, E. P. (1999). Executive functioning in children with heavy prenatal alcohol exposure. Alcohol. Clin. Exp. Res. 23, 1808-1815.

Melcer, T., Gonzalez, D., Barron, S., and Riley, E. P. (1994). Hyperactivity in pre-weanling rats following postnatal alcohol exposure. Alcohol 11, 41-45.

Miller, L. J., Coll, J. R., and Schoen, S. A. (2007).A randomized controlled pilot study of the effectiveness of occupational therapy for children with sensory modulation disorder. Am. J. Occup. Ther. 61, 228-238.

Miller, L. J., McIntosh, D. N., McGrath, J., Shyu, V., Lampe, M., Taylor, A. K., Tassone, F., Neitzel, K., Stackhouse, T., and Hagerman, R. J. (1999). Electrodermal responses to sensory stimuli in individuals with fragile $\mathrm{X}$ syndrome: A preliminary report. Am. J. Med. Genet. 83, 268-279.

Moore, C. F., Gajewski, L. L., Laughlin, N. K., Luck, M. L., Larson, J. A., and Schneider,M.L.(2008).Developmental lead exposure induces tactile defensiveness in rhesus monkeys (Macaca mulatta). Environ. Health Perspect. 116, 1322-1326.

Morse, B. A., and Cermak, S. A. (1994). Sensory integration in children with FAS [Abstract 502]. Alcohol Clin. Exp. Res. 18, 503.

Mukherjee, J., Yang, Z. Y., Das, M. K., and Brown, T. (1995). Fluorinated benzamide neuroleptics-III development of (S)-N-[(1-allyl-2-pyrrolidinyl)methyl]5-(3-[18F]fluoropropyl0-2,3-dimethoxybenzamide as an improved dopamine D-2 receptor tracer. Nucl. Med. Biol. 22, 283-296.

Mukherjee, J., Yang, Z. Y., Lew, R., Brown, T., Kronmal, S., Cooper, M. D., and Seiden, L. S. (1997). Evaluation of damphetamine effects on the binding of dopamine $\mathrm{D} 2$ receptor radioligand, $18 \mathrm{~F}$-fallypride in nonhuman primates using positron emission tomography. Synapse 27, 1-13.

Murphy, D. L., Lerner, A., Rudnick, G., and Lesch, K. P. (2004). Serotonin transporter: gene, genetic disorders, and pharmacogenetics. Mol. Interv. 4, 109-123.

Newell-Morris, L., and Fahrenbruch, C. E. (1985). Practical and evolutionary considerations for use of the nonhuman primate model in prenatal research. In Nonhuman Primate Models for Human Growth and Development, E. S. Watts, ed. (New York, Liss), pp. 9-40.

Parush, S., Sohmer, H., Steinberg, A., and Kaitz, M. (2007). Somatosensory function in boys with ADHD and tactile defensiveness. Physiol. Behav. 90, 553-558.

Rakic, P. (1985). Limits of neurogenesis in primates. Science 227, 154-156.

Rakic, P. (1988). Defects of neuronal migration and the pathogenesis of cortical malformations. Prog. Brain Res. 73, 15-37.

Rakic, P. (1995). Development of cerebral cortex in human and nonhuman primates. In Child and Adolescent Psychiatry, M. Lewis, ed. (Baltimore, Williams \& Wilkins), pp. 9-29.
Ramadoss, J.,Lunde,E.R.,Chen, W. J., West, J. R., and Cudd, T. A. (2007). Temporal vulnerability of fetal cerebellar Purkinje cells to chronic binge alcohol exposure: ovine model. Alcohol. Clin. Exp. Res. 31, 1738-1745.

Redgrave, P., Prescott, T. J., and Gurney, K. (1999). Is the short-latency dopamine response too short to signal reward error? Trends Neurosci. 22, 146-151.

Richardson, G. A., Day, N. L., and Taylor, P. M. (1989). The effect of prenatal alcohol, marijuana, and tobacco exposure on neonatal behavior. Infant Behav. Dev. 12, 199-209.

Ridley, M. (2003). Nature via Nuture: Genes, Experience and What Makes us Human. London, Fourth Estate.

Roberts, A. D., Moore, C. F., DeJesus, O. T., Barnhart, T.E., Larson, J.A., Mukherjee, J., Nickles, R. J., Schueller, M. J., Shelton, S. E., and Schneider, M. L. (2004). Prenatal stress, moderate fetal alcohol, and dopamine system function in rhesus monkeys. Neurotoxicol. Teratol. 26, 169-178.

Rutter, M., Moffitt, T. E., and Caspi, A. (2006). Gene-environment interplay and psychopathology: multiple varieties but real effects. J. Child. Psychol. Psychiatry 47, 226-261.

Schaaf, R. C., and Nightlinger, K. M. (2007). Occupational therapy using a sensory integrative approach: a case study of effectiveness. Am. J. Occup. Ther. 61, 239-246.

Schneider, M. L., Moore, C. F., Barnhart, T. E., Larson, J. A., DeJesus, O. T., Mukherjee, J., Nickles, R. J., Converse, A. K., Roberts, A. D., and Kraemer, G. W. (2005). Moderate-level prenatal alcohol exposure alters striatal dopamine system function in rhesus monkeys. Alcohol. Clin. Exp. Res. 29, 1685-1697.

Schneider, M. L., Moore, C. F., Gajewski, L. L.,Larson, J.A., Roberts, A.D., Converse, A.K., and DeJesus, O.T. (2008). Sensory processing disorder in a primate model: evidence from a longitudinal study of prenatal alcohol and prenatal stress effects. Child Dev. 79, 100-113.

Shen, R. Y., Hannigan, J. H., and Kapatos, G. (1999).Prenatal ethanol reduces the activity of adult midbrain dopamine neurons. Alcohol. Clin. Exp. Res. 23, 1801-1807.

Shetty, A. K., Burrows, R. C., and Phillips, D. E. (1993). Alterations in neuronal development in the substantia nigra pars compacta following in utero ethanol exposure: Immunohistochemical and Golgi studies. Neuroscience 52, 311-322.

Smith, G. N., Brien, J. F., Carmichael, L., Homan, J., Clarke, D. W., and Patrick, J. (1989). Development of tolerance to ethanol-induced suppression of breathing movements and brain activity in the near-term fetal sheep during short-term maternal administration of ethanol. J. Dev. Physiol. 11, 189-197.

Sulik, K. K., Johnston, M. C., and Webb, M. A. (1981). Fetal alcohol syndrome: Embryogenesis in a mouse model. Science 214, 936-938.

Volkow, N. D., Fowler, J. S., Wang, G. J., and Goldstein, R. Z. (2002). Role of dopamine, the frontal cortex and memory circuits in drug addiction: insight from imaging studies. Neurobiol Learn Mem 78, 610-624.

West, J. R., Dewey, S. L., Pierce, D. R., and Black, A. C. Jr. (1984). Prenatal and early postnatal exposure to ethanol permanently alters the rat hippocampus. Ciba Found. Symp. 105, 8-25.

Zecevic, N., and Rakic, P. (1991). Synaptogenesis in monkey somatosensory cortex. Cereb. Cortex 1, 510-523.

Conflict of Interest Statement: The authors declare that the research was conducted in the absence of any commercial or financial relationships that could be construed as a potential conflict of interest.

Received: 10 April 2009; paper pending published: 11 August 2009; accepted: 23 October 2009; published online: 10 November 2009.

Citation: Schneider ML, Moore CF, Larson $J A$, Barr CS, DeJesus OT and Roberts AD (2009) Timing of moderate level prenatal alcohol exposure influences gene expression of sensory processing behavior in rhesus monkeys. Front. Integr. Neurosci. 3:30. doi: 10.3389/neuro.07.030.2009

Copyright () 2009 Schneider, Moore, Larson, Barr, DeJesus and Roberts. This is an open-access article subject to an exclusive license agreement between the authors and the Frontiers Research Foundation, which permits unrestricted use, distribution, and reproduction in any medium, provided the original authors and source are credited. 